\title{
DOE-Imaging grant FG02-06ER15829, entitled "Developing Laser-Induced Re-Collision Electron Self-Diffraction" - Brief summary of accomplishments
}

Our principal goal was the experimental demonstration of Laser-Induced Electron Diffraction (LIED). We started with measuring 3D electron momenta spectra in aligned nitrogen and oxygen molecules. Chakra Maharjan (Ph.D. student of Lew Cocke) was a lead researcher on this project. Although Chakra succeeded in obtaining those spectra, we were scooped by the publication of identical results in Science by the NRC Ottawa group [1]. Our results were never published as a refereed article, but became a part of Chakra's Ph.D. dissertation [2]. That Science paper was the first experimental demonstration of Laser-Induced Electron Diffraction (LIED). Chakra also worked on wavelength dependence of 3D ATI spectra of atoms and molecules using tunable OPA pulses [3].

Another Ph.D. student, Maia Magrakvelidze (her GRA was funded by the grant), started working on COLTRIMS experiments using OPA pulses (1800 nm wavelength). After some initial experiments it became apparent that COLTRIMS did not yield sufficient count rates of electrons in the high-energy part of the spectrum to see diffraction signatures with acceptable statistics (unfavorable scaling of the electron yield with laser wavelength was partly to blame). Nevertheless, Maia managed to use COLTRIMS and OPA to measure the angular dependence of the tunneling ionization rate in $\mathrm{D}_{2}$ molecules [4].

Following the initial trial experiments, the decision was made to switch from COLTRIMS to VMI in order to increase the count rates by a factor of $\sim 100$, which may have given us a chance to see LIED. Research Associate Dr. Sankar De (his salary was funded by the grant), in collaboration with Matthias Kling's group (then at MPQ Garching), proceeded to design a special multi-electrode VMI spectrometer for capturing high-energy ATI electrons and to install it in place of COLTRIMS inside our experimental chamber. That apparatus was later used for the first demonstration of field-free orientation in $\mathrm{CO}$ using two-color laser pulses [5] as well as for a series of other experiments, such as pump-probe studies of molecular dynamics with few-cycle laser pulses [6,7], control of electron localization in dissociating hydrogen molecules using twocolor laser pulses [8], and ATI spectra of Xe ionized by two-color laser pulses [9].

In parallel, Dipanwita Ray (Ph.D. student of Lew Cocke) worked on measuring angle-resolved ATI spectra of noble gases using a stereo-ATI phasemeter as a TOF electron spectrometer. She observed the angular diffraction structures in 3D ATI spectra of Ar, Kr and Xe [10], which were interpreted in terms of the Quantitative Rescattering theory newly developed by C.D. Lin.

We also attempted to use a much more powerful OPA (five times more energy per pulse than the one we had at JRML) available at the Advanced Laser Light Source (ALLS) in Montreal to observe LIED. Two visits to ALLS by the PI, Igor Litvinyuk, and one visit by the PI's Ph.D. student (Irina Bocharova) were funded by the grant. Though we failed to observe LIED (the repetition rate of the ALLS OPA was too low at only $100 \mathrm{~Hz}$ ), this international collaboration resulted in several publications on other related subjects, such as the wavelength dependence of laser Coulomb explosion of hydrogen [11], the wavelength dependence of non-sequential double ionization of neon and argon [12], the demonstration of charge-resonance enhanced ionization in 
$\mathrm{CO}_{2}$ [13], and the study of non-elastic scattering processes in $\mathrm{H}_{2}$ [14]. Theoretical efforts to account for the hydrogen Coulomb explosion experiment resulted in another paper by Maia Magrakvelidze as lead author [15].

Although for various reasons we failed to achieve our main goal of observing LIED, we salute the recent success in this endeavor by Lou DiMauro's group (with theoretical support from our KSU colleague C.D. Lin) published in Nature [16], which validates our approach.

\section{References (papers marked with • present research fully or partially funded by the grant)}

1. M. Meckel et al., "Laser Induced Electron Tunneling and diffraction”, Science 320, 1478-1482 (2008). http://www.sciencemag.org/content/320/5882/1478.short

2. C.M. Maharjan, "Momentum Imaging Studies of Electron and Ion Dynamics in a Strong Laser Field”, Ph.D. Dissertation (Kansas State University, 2007)

- $\underline{\text { http://jrm.phys.ksu.edu/theses.html }}$

3. C.M. Maharjan, A.S. Alnaser, I. Litvinyuk, P. Ranitovic, and C.L. Cocke, "Wavelength dependence of momentum-space images of low-energy electrons generated by short intense laser pulses at high intensities”, J. Phys. B - At. Mol. Opt. Phys. 39, 1955 (2006).

- http://stacks.iop.org/JPhysB/39/1955

4. M. Magrakvelidze, F. He, S. De, I. Bocharova, D. Ray, U. Thumm, and I.V. Litvinyuk, "Angular dependence of the strong-field ionization measured in randomly oriented hydrogen molecules", Phys. Rev. A 79, 033408 (2009).

- http://pra.aps.org/pdf/PRA/v79/i3/e033408

5. S. De, I. Znakovskaya, D. Ray, F. Anis, Nora G. Johnson, I.A. Bocharova, M. Magrakvelidze, B.D. Esry, C.L. Cocke, I.V. Litvinyuk, and M.F. Kling, "Field-Free Orientation of CO Molecules by Femtosecond Two-Color Laser Fields", Phys. Rev. Lett. 103, 153002 (2009).

- http://prl.aps.org/pdf/PRL/v103/i15/e153002

6. S. De, I.A. Bocharova, M. Magrakvelidze, D. Ray, W. Cao, B. Bergues, U. Thumm, M.F. Kling, I.V. Litvinyuk, and C.L. Cocke, "Tracking nuclear wave-packet dynamics in molecular oxygen ions with few-cycle infrared laser pulses”, Phys. Rev. A 82, 013408 (2010).

- $\underline{\text { http://pra.aps.org/abstract/PRA/v82/i1/e013408 }}$

7. S. De, M. Magrakvelidze, I.A. Bocharova, D. Ray, W. Cao, I. Znakovskaya, H. Li, Z. Wang, G. Laurent, U. Thumm, M.F. Kling, I.V. Litvinyuk, I. Ben-Itzhak, and C.L. Cocke, "Following dynamic nuclear wave packets in $\mathrm{N}_{2}, \mathrm{O}_{2}$, and $\mathrm{CO}$ with few-cycle infrared pulses", Phys. Rev. A 84, 043410 (2011).

- http://pra.aps.org/abstract/PRA/v84/i4/e043410

8. D. Ray, F. He, S. De, W. Cao W, H. Mashiko, P. Ranitovic, K.P. Singh, I. Znakovskaya, U. Thumm, G.G. Paulus, M.F. Kling, I.V. Litvinyuk, and C.L. Cocke, "Ion-Energy Dependence of Asymmetric Dissociation of D-2 by a Two-Color Laser Field", Phys. Rev. Lett. 103, 223201 (2009).

- http://prl.aps.org/pdf/PRL/v103/i22/e223201

9. D. Ray, Zhangjin Chen, S. De, W. Cao, I.V. Litvinyuk, A.T. Le, C.D. Lin, M.F. Kling, and C.L. Cocke, "Momentum spectra of electrons rescattered from rare-gas targets following their extraction by one- and two-color femtosecond laser pulses", Phys. Rev. A 83, 013410 (2011).

- http://pra.aps.org/abstract/PRA/v83/i1/e013410 
10. D. Ray, B. Ulrich, I. Bocharova, C. Maharjan, P. Ranitovic, B. Gramkow, M. Magrakvelidze, S. De, I.V. Litvinyuk, A.T. Le, T. Morishita, C.D. Lin, G.G. Paulus, and C.L. Cocke, "Large-angle electron diffraction structure in laser-induced rescattering from rare gases", Phys. Rev. Lett. 100, 143002 (2008).

- $\underline{\text { http://link.aps.org/doi/10.1103/PhysRevLett.100.143002 }}$

11. I.V. Litvinyuk, A.S. Alnaser, D. Comtois, D. Ray, A.T. Hasan, J.-C. Kieffer, D.M. Villeneuve, "Wavelength-dependent study of strong-field Coulomb explosion of hydrogen", New J. Phys. 10, 083011 (2008).

- $\underline{\text { http://www.iop.org/EJ/article/-search=60218028.1/1367-2630/10/8/083011/njp8 } 8083011 . h t m l}$

12. A.S. Alnaser, D. Comtois, A.T. Hasan, D.M. Villeneuve, J.-C. Kieffer, and I.V. Litvinyuk, "Strongfield non-sequential double ionization: wavelength dependence of ion momentum distributions for neon and argon", J. Phys. B: At. Mol. Opt. Phys. 41, 031001 (2008).

- http://iopscience.iop.org/0953-4075/41/3/031001

13. I. Bocharova, R. Karimi, E. F. Penka, J.-P. Brichta, P. Lassonde, X. Fu, J.-C. Kieffer, A. D. Bandrauk, I. Litvinyuk, J. Sanderson, and F. Légaré, "Charge Resonance Enhanced Ionization of $\mathrm{CO}_{2}$ Probed by Laser Coulomb Explosion Imaging”, Phys. Rev. Lett. 107, 063201 (2011).

- http://link.aps.org/doi/10.1103/PhysRevLett.107.063201

14. F. Legare, I. A. Bocharova, P. Lassonde, R. Karimi, J. H. Sanderson, T. Johnston, J.-C. Kieffer and I. V Litvinyuk, "Inelastic rescattering processes in molecules measured with few-cycle laser pulses", J. Phys. B-At. Mol. Opt. Phys. 42, 235601 (2009).

- $\underline{\text { http://stacks.iop.org/JPhysB/42/235601 }}$

15. M. Magrakvelidze, F. He, T. Niederhausen, I. V. Litvinyuk, U. Thumm, "Quantum-beat imaging of the nuclear dynamics in $\mathrm{D}_{2}^{+}$: Dependence of bond softening and bond hardening on laser intensity, wavelength, and pulse duration”, Phys. Rev. A 79, 033410 (2009).

- http://pra.aps.org/pdf/PRA/v79/i3/e033410

16. C.I. Blaga et al., "Imaging ultrafast molecular dynamics with laser-induced electron diffraction", Nature 483, 194-197 (2012). http://www.nature.com/nature/journal/v483/n7388/full/nature10820.html 


\section{Publications fully or partially funded by this grant}

1. C.M. Maharjan, "Momentum Imaging Studies of Electron and Ion Dynamics in a Strong Laser Field”, Ph.D. Dissertation (Kansas State University, 2007) http://jrm.phys.ksu.edu/theses.html

2. C.M. Maharjan, A.S. Alnaser, I. Litvinyuk, P. Ranitovic, and C.L. Cocke, "Wavelength dependence of momentum-space images of low-energy electrons generated by short intense laser pulses at high intensities”, J. Phys. B - At. Mol. Opt. Phys. 39, 1955 (2006).

http://stacks.iop.org/JPhysB/39/1955

3. M. Magrakvelidze, F. He, S. De, I. Bocharova, D. Ray, U. Thumm, and I.V. Litvinyuk, "Angular dependence of the strong-field ionization measured in randomly oriented hydrogen molecules", Phys. Rev. A 79, 033408 (2009). http://pra.aps.org/pdf/PRA/v79/i3/e033408

4. S. De, I. Znakovskaya, D. Ray, F. Anis, Nora G. Johnson, I.A. Bocharova, M. Magrakvelidze, B.D. Esry, C.L. Cocke, I.V. Litvinyuk, and M.F. Kling, "Field-Free Orientation of CO Molecules by Femtosecond Two-Color Laser Fields”, Phys. Rev. Lett. 103, 153002 (2009). http://prl.aps.org/pdf/PRL/v103/i15/e153002

5. S. De, I.A. Bocharova, M. Magrakvelidze, D. Ray, W. Cao, B. Bergues, U. Thumm, M.F. Kling, I.V. Litvinyuk, and C.L. Cocke, "Tracking nuclear wave-packet dynamics in molecular oxygen ions with few-cycle infrared laser pulses”, Phys. Rev. A 82, 013408 (2010).

http://pra.aps.org/abstract/PRA/v82/i1/e013408

6. S. De, M. Magrakvelidze, I.A. Bocharova, D. Ray, W. Cao, I. Znakovskaya, H. Li, Z. Wang, G. Laurent, U. Thumm, M.F. Kling, I.V. Litvinyuk, I. Ben-Itzhak, and C.L. Cocke, "Following dynamic nuclear wave packets in $\mathrm{N}_{2}, \mathrm{O}_{2}$, and $\mathrm{CO}$ with few-cycle infrared pulses", Phys. Rev. A 84, 043410 (2011).

http://pra.aps.org/abstract/PRA/v84/i4/e043410

7. D. Ray, F. He, S. De, W. Cao W, H. Mashiko, P. Ranitovic, K.P. Singh, I. Znakovskaya, U. Thumm, G.G. Paulus, M.F. Kling, I.V. Litvinyuk, and C.L. Cocke, "Ion-Energy Dependence of Asymmetric Dissociation of D-2 by a Two-Color Laser Field", Phys. Rev. Lett. 103, 223201 (2009). http://prl.aps.org/pdf/PRL/v103/i22/e223201

8. D. Ray, Zhangjin Chen, S. De, W. Cao, I.V. Litvinyuk, A.T. Le, C.D. Lin, M.F. Kling, and C.L. Cocke, "Momentum spectra of electrons rescattered from rare-gas targets following their extraction by one- and two-color femtosecond laser pulses", Phys. Rev. A 83, 013410 (2011). http://pra.aps.org/abstract/PRA/v83/i1/e013410

9. D. Ray, B. Ulrich, I. Bocharova, C. Maharjan, P. Ranitovic, B. Gramkow, M. Magrakvelidze, S. De, I.V. Litvinyuk, A.T. Le, T. Morishita, C.D. Lin, G.G. Paulus, and C.L. Cocke, "Large-angle electron diffraction structure in laser-induced rescattering from rare gases", Phys. Rev. Lett. 100, 143002 (2008). http://link.aps.org/doi/10.1103/PhysRevLett.100.143002

10. I.V. Litvinyuk, A.S. Alnaser, D. Comtois, D. Ray, A.T. Hasan, J.-C. Kieffer, D.M. Villeneuve, "Wavelength-dependent study of strong-field Coulomb explosion of hydrogen", New J. Phys. 10, 083011 (2008). http://www.iop.org/EJ/article/-search=60218028.1/1367-2630/10/8/083011/njp8 8 083011.html

11. A.S. Alnaser, D. Comtois, A.T. Hasan, D.M. Villeneuve, J.-C. Kieffer, and I.V. Litvinyuk, "Strongfield non-sequential double ionization: wavelength dependence of ion momentum distributions for neon and argon", J. Phys. B: At. Mol. Opt. Phys. 41, 031001 (2008).

http://iopscience.iop.org/0953-4075/41/3/031001 
12. I. Bocharova, R. Karimi, E. F. Penka, J.-P. Brichta, P. Lassonde, X. Fu, J.-C. Kieffer, A. D. Bandrauk, I. Litvinyuk, J. Sanderson, and F. Légaré, "Charge Resonance Enhanced Ionization of $\mathrm{CO}_{2}$ Probed by Laser Coulomb Explosion Imaging”, Phys. Rev. Lett. 107, 063201 (2011). http://link.aps.org/doi/10.1103/PhysRevLett.107.063201

13. F. Legare, I. A. Bocharova, P. Lassonde, R. Karimi, J. H. Sanderson, T. Johnston, J.-C. Kieffer and I. V Litvinyuk, "Inelastic rescattering processes in molecules measured with few-cycle laser pulses", J. Phys. B-At. Mol. Opt. Phys. 42, 235601 (2009).

http://stacks.iop.org/JPhysB/42/235601

14. M. Magrakvelidze, F. He, T. Niederhausen, I. V. Litvinyuk, U. Thumm, "Quantum-beat imaging of the nuclear dynamics in $\mathrm{D}_{2}^{+}$: Dependence of bond softening and bond hardening on laser intensity, wavelength, and pulse duration”, Phys. Rev. A 79, 033410 (2009).

http://pra.aps.org/pdf/PRA/v79/i3/e033410

15. M. Zohrabi, J. McKenna, B. Gaire, Nora G. Johnson, K. D. Carnes, S. De, I. A. Bocharova, M. Magrakvelidze, D. Ray, I. V. Litvinyuk, C. L. Cocke, and I. Ben-Itzhak, "Vibrationally-resolved structure in $\mathrm{O}_{2}{ }^{+}$dissociation induced by intense ultrashort laser pulses" Physical Review A 83, 053405 (2011).

http://pra.aps.org/abstract/PRA/v83/i5/e053405

\section{Invited talks and contributed conference presentations by I.V. Litvinyuk reporting on research funded by the grant}

\section{Invited talks}

"Controlling ultrafast processes in molecules with intense two-color laser pulses", University of Tokyo, Seminar at the Institute for Solid State Physics, Kashiwa, Japan, 17 May 2010.

"Controlling ultrafast processes in molecules with intense two-color laser pulses", POSTECH colloquium, Pohang, Korea, 19 May 2010.

"Controlling ultrafast processes in molecules with intense two-color laser pulses", INRS Quebec Seminar, Montreal, Canada, 15 June 2010.

"Controlling ultrafast processes in molecules with intense two-color laser pulses", NRC Ottawa Seminar, Ottawa, Canada, 29 June 2010.

"Coulomb explosion imaging of nuclear wave packet dynamics induced by few-cycle laser pulses in diatomic molecules", $9^{\text {th }}$ Asian International Seminar on Atomic and Molecular Physics (AISAMP 9), Seoul, Korea, 4 October 2010.

"Making molecular movies", Public lecture directed to high-school and first year undergraduate students, Griffith University, Nathan, Queensland, 26 May 2011.

"Dynamics and control of electron localization in dissociating molecules", Symposium on Attosecond Science and Technology, Korean Physical Society Meeting, Daejoeon, Korea, to be presented on 26 April 2012.

\section{Conference presentations}

"Real-time dynamics of electron localization observed in dissociating $\mathrm{N}_{2}{ }^{3+}$, DAMOP, Charlottesville, Virginia, May 19-23, 2009.

"Dynamic field-free orientation of polar molecules by intense two-color femtosecond laser pulses", $18^{\text {th }}$ Annual International Workshop on Laser Physics, Barcelona, July 13-17, 2009

"Real-time dynamics of electron localization observed in dissociating molecules", ACOLS ACOFT 09, Adelaide, South Australia 29 Nov - 3 Dec 2009. 
"Effects of laser pulse duration and intensity on Coulomb explosion of $\mathrm{CO}_{2}$ : Signatures of charge-resonance enhanced ionization", Gordon Multiphoton Conference, Tilton, New Hampshire, 6-10 June 2010

"Coulomb explosion imaging of nuclear wave packet dynamics induced by few-cycle laser pulses in diatomic molecules", $9^{\text {th }}$ Asian International Seminar on Atomic and Molecular Physics (AISAMP 9), Seoul, Korea, 4 October 2010.

"Charge Resonance Enhanced Ionization of $\mathrm{CO}_{2}$ probed by Coulomb explosion imaging with laser pulses of varying duration", $19^{\text {th }}$ AIP Congress, Melbourne, Victoria, 5-9 Dec 2010.

"Enhanced Ionization in Tri-Atomic Molecules Observed with Coulomb Explosion Imaging", ICOMP12, Sapporo, Japan, 3-6 July 2011

"Enhanced Ionization in Di- and Tri-Atomic Molecules Observed with Coulomb Explosion Imaging", IQEC/CLEO PR, Sydney, New South Wales, 28 Aug - 1 Sep 2011

\section{Invited talks and contributed conference presentations by Dr. Sankar De reporting on research funded by the grant}

\section{Invited talks}

"Probing ultrafast molecular dynamics using a pump-probe approach", Applied Nuclear Physics Division, Saha Institute of Nuclear Physics, Kolkata, India, 12 October, 2010.

\section{Conference presentations}

"Following dynamic nuclear wave packets in $\mathrm{N}_{2}, \mathrm{O}_{2}$ and $\mathrm{CO}$ with few-cycle infrared pulses", CDAMOP, Delhi, India, 14-16 December, 2011.

"Tracking dynamic wave packets in the $\mathrm{O}_{2}$ dication using a pump/probe approach", DAMOP, Houston, USA, 25-29 May, 2010.

"Dynamic field-free orientation of polar molecules by intense two-color femtosecond laser pulses", ATTO-09, Manhattan, Kansas, USA, 28 July - 1 August, 2009.

"Dynamic field-free orientation of polar molecules by intense two-color femtosecond laser pulses", DAMOP, Charlottesville, Univ. of Virginia, USA, May 19-23, 2009.

"A multi-electrode velocity-map imaging apparatus to study laser induced molecular dynamics", DAMOP, Charlottesville, Univ. of Virginia, USA, May 19-23, 2009.

\section{Contributed conference presentations by graduate student reporting on research funded by the grant}

\section{Maia Magrakvelidze}

"Electron-ion momentum coincidence experiments on hydrogen molecules dissociated by intense femtosecond laser pulses", DAMOP, Charlottesville, Virginia, May 19-23, 2009.

"Quantum beat imaging of the nuclear dynamics in $\mathrm{D}_{2}{ }^{+}$: Dependence of bond softening and hardening on laser intensity, wavelength and pulse duration”, DAMOP, Charlottesville, Virginia, May 19-23, 2009.

\section{Irina A. Bocharova}

"Time-resolved Laser Coulomb Explosion Imaging of $\mathrm{N}_{2}, \mathrm{O}_{2}$ and $\mathrm{CO}$ molecular dynamics", DAMOP, Charlottesville, Virginia, May 19-23, 2009. 